\title{
FORMATION OF OXIDIZED ALUMINUM NANOPOWDERS BY EXPOSING ALUMINUM TO A SERIES OF DOUBLE LASER PULSES IN AIR
}

\author{
Kh. Bazzal, ${ }^{\text {a }}$ N. A. Alekseenko, ${ }^{\text {b }}$ E. S. Voropay, \\ M. N Kovalenko, ${ }^{\text {a }}$ N. H. Trinh, ${ }^{\text {c }}$ and A. P. Zazhogin ${ }^{a}{ }^{*}$
}

UDC 533.9.082.5;621.373.826;621.793.79

The effects of the interval between pulses and the number of double laser pulses on the component and charge composition of the laser plasma formed by these pulses acting on an AD1 aluminum alloy target (LSS-1 spectrometer) are studied. It is shown that the concentration of Al ions and the products of their interaction with atmospheric $\mathrm{O}_{2}$ and $N_{2}$ increase by several orders of magnitude if an interval between pulses of 7-16 $\mu$ s rather than a zero interval is used. Formation of Al ions is studied as a function of pulse energy with an interval between pulses of $10 \mu$ s. The strongest lines for Al III ions are observed for a small number ( 15) consecutive double pulses in series and a pulse energy of $53 \mathrm{~mJ}$. The intensity of the lines for Al III ions decreases by about five times if the number of pulses in the series increases above a certain level (10-20) because of their active interaction with atmospheric molecules and atoms at the exit of the micronozzle when the channel shape is changed. Al nanopowders coated with a layer of aluminum oxide produced by irradiating an Al target with a series of 15 double laser pulses per point have sizes of mainly 50-60 $\mu \mathrm{m}$.

Keywords: oxidized nanopowders of $\mathrm{Al}, \mathrm{Al}_{2} \mathrm{O}_{3}$, AlO suboxides, pulsed laser sputtering, laser plasma, laser spark spectrometry, multicharged ions, double laser pulses.

Introduction. Intermediate energy sources that enable expenses for ecological conservation and the rational redistribution of energy to be reduced are one potential possibility for storing energy. Currently, the search for new solutions for improving the efficiency, economics, and reliability of alternative energy sources and reducing capital and operating costs etc. has been prompted by the fact that the specific economic parameters of alternative energy sources are inferior to the analogous parameters of traditional ones. Mankind will soon be forced to use renewable energy sources that, as a rule, are located at significant distances from traditional population centers and sites of energy utilization. Effective implementation of renewable energy sources requires technology development for storage of the produced energy and methods for transporting it to consumers [1].

Aluminum nanopowders (NPs) could be one of the most promising materials for solving these problems. It is noteworthy that metal NPs are chemically more reactive than roughly disperse and micron-sized powders. Metal NPs prepared by any known method in $\mathrm{Ar}, \mathrm{H}_{2}$, or a mixture of these gases are pyrophoric and ignite on contact with air. For example, A NPs react explosively with $\mathrm{H}_{2} \mathrm{O}$ at $80^{\circ} \mathrm{C}$, i.e., exhibit the properties of alkali metals. Therefore, passivation of the particle surface is a necessary step in metal NP production technology [1-4].

Metal NPs are used as reagents, components, and sintering additives to manufacture ceramic materials because of their special properties such as significantly lower sintering temperatures and melting points than ordinary disperse materials and higher chemical reactivity for oxidation [3-5]. A study of Al NPs as a sintering activator for ASD-4 industrial powder found that the sintering temperature decreased (to $450-500^{\circ} \mathrm{C}$ ), the microhardness increased (from $325 \mathrm{MPa}$ for ASD-4 to $450 \mathrm{MPa}$ for ASD-4 + Al-NP), and the degree of shrinkage increased up to $60 \%$ as the mass fraction of Al-NPs increased [5].

\footnotetext{
*To whom correspondence should be addressed.
}

${ }^{\mathrm{a}}$ Belarusian State University, Minsk, 220030, Belarus; email: zajogin_an@mail.ru; ${ }^{\mathrm{b}}$ Powder Metallurgy Institute Named after Academician O. V. Roman, Minsk, 220005, Belarus; email: alekseenkon@rambler.ru; ${ }^{\mathrm{c}}$ Vinh University, Vinh, Vietnam; email: ngochoangch10@gmail.com. Translated from Zhurnal Prikladnoi Spektroskopii, Vol. 88, No. 1, pp. 96-103, January-February, 2021. Original article submitted October 8, 2020. 
A combined additive containing metallic $\mathrm{Al}$ and its oxide gave good results if used as an $\mathrm{Al}_{2} \mathrm{O}_{3}-\mathrm{Al}$ system [3-5]. This combination enabled the production of a rather dense ceramic without adding extraneous impurities. An unstabilized $\mathrm{ZrO}_{2}$ sintered ceramic could be produced by adding Al-NPs ( $\geq 10$ mass $\%$ ) to the starting $\mathrm{ZrO}_{2}-\mathrm{NPs}$ [4].

The most universal methods for preparing NPs with the minimal contamination with respect to versatility of the used target material (large powders and their mixtures, metals and alloys, mixtures of metal and nonmetals) are vaporization by laser radiation [6-8] and electron beams [9]. An advantage of using laser radiation to vaporize most materials is the small penetration depth of the beam into the material, which allows the target to be vaporized with relatively small energy losses. Both continuous [6] and pulsed lasers with various pulse lengths [7, 8] are used to vaporize targets.

The component, charge, and energy distribution of a laser plume can be analyzed and intentionally changed by acting on the primary plasma with additional laser radiation. Double-pulse laser action at different beam incident angles on the target and plasma enabled simultaneous highly sensitive spectral analysis, production and control of the concentrations of excited and charged plasma particles, and control of the composition of the plasma directed at the substrate [10, 11].

The goal of the present work was to demonstrate the possibility and determine the conditions for producing AlNPs by ablation of Al targets using series of double laser pulses in air. The studies are crucial for improving the production technology of metal NPs for alternative energy sources.

Experimental. An LSS-1 laser single-channel atomic emission spectrometer (SP LOTIS TII) with pulse repetition rate up to $10 \mathrm{~Hz}$ at $\lambda=1064 \mathrm{~nm}$ and pulse length $\sim 15 \mathrm{~ns}$ was used for the studies. The time shift between double pulses was $0-100 \mu \mathrm{s}$ in steps of $1 \mu \mathrm{s}$. The size of the focused spot was $\sim 50 \mu \mathrm{m}$ with an objective focal distance of $104 \mathrm{~mm}$.

The formation kinetics of the reaction products of $\mathrm{Al}$ atoms and oxygen radical, i.e., $\mathrm{AlO}$, and $\mathrm{AlN}$ nanocomplexes were studied using the emission spectra of these molecules. The strongest vibronic bands in emission spectra of AlO were those with $\lambda=484.21$ and $486.5 \mathrm{~nm}$; of AlN (507.8 nm), lines of ions Al II (466.3), Al III (452.92), and N II (399.5). The Al target made of AD1 was placed in a closed rectangular glass cuvette $(40 \times 20 \times 30 \mathrm{~mm})$. Products that settled on the surface of silicon plates installed outside the laser plume zone (on the bottom and one side of the cuvette) were analyzed using a MIRA3 high-resolution scanning electron microscope (SEM) with an EDX X-Max x-ray spectral microanalyzer.

The experiments found that an interval between pulses of 7-16 $\mu$ s gave the strongest ion lines and molecular bands [11-13]. Figure 1a shows dependences of the intensities of $\mathrm{Al}$ and $\mathrm{N}$ ion lines and $\mathrm{AlN}$ nanocluster and $\mathrm{AlO}$ radical bands on the interval between pulses using 40 pulses per point and pulse energy $37 \mathrm{~mJ}$. Next, the experiments used an interval between laser pulses of $10 \mu \mathrm{s}$. Figure $1 \mathrm{~b}$ illustrates the formation of $\mathrm{Al}$ and $\mathrm{N}$ ions, AlN nanoclusters, and $\mathrm{AlO}$ radicals as functions of pulse energy using 40 pulses per point. Figure 1c plots the intensities of these lines and bands for each pulse number for the first 25 pulses with pulse energy $53 \mathrm{~mJ}$. Figure 1a shows that the concentration of $\mathrm{Al}$ ions and the products of their reaction with atmospheric $\mathrm{O}_{2}$ and $\mathrm{N}_{2}$ increased by several orders of magnitude for an interval between pulses of 7-16 $\mu \mathrm{s}$ as compared with no interval. The formation of Al III, AlN nanoclusters, and AlO radicals increased rapidly with increasing energy to $35-40 \mathrm{~mJ}$ and then leveled off. However, the intensity of the Al II line continued to increase. Figure $1 \mathrm{c}$ shows a good correlation between the increase in the $\mathrm{AlO}$ and $\mathrm{AlN}$ band intensities and a substantial decrease in the $\mathrm{Al}$ III line intensity $(452.92 \mathrm{~nm})$. However, the line intensity of Al II ions $(466.3 \mathrm{~nm})$ increased in steps. Results obtained with pulse energy $53 \mathrm{~mJ}$ (Fig. 1c) showed that the formation rate of Al nanoclusters and products of their reaction with $\mathrm{O}_{2}$ and $\mathrm{N}_{2}$ depended very strongly on the number of consecutive double pulses (microchannel depth). The sharp time threshold in the formation rate of $\mathrm{AlO}$ radicals was indicative of the plasma formation specifics within the resulting rather deep microchannel [6-8].

The Al III ion line was strongest in the range of 1-15 pulses while AlO suboxide and AlN nanoclusters started to form effectively after 7-10 pulses. Significantly less products formed after fewer (0-7) pulses. This could be explained by effective removal of the particles from the still shallow crater because of convective air currents.

Based on these data, a pulse energy of $53 \mathrm{~mJ}$ and an interval between pulses of $10 \mu$ s were used to evaluate the ability for practical production of Al NPs. The irradiation occurred in series of 15 double pulses per point for $10 \mathrm{~min}$. The total number of points was 100 in an area of $4 \times 4 \mathrm{~mm}^{2}$. Figure 2 shows SEM images of NPs and NP agglomerates that were synthesized using double laser pulses on an AD1 Al alloy target. The size of the primary particles was estimated as 50-60 nm using high-resolution electron microscopy. Particles that gathered into agglomerates had a crystalline structure and spherical shape.

The spherical shape of NPs produced under extreme conditions indicated that they were liquefied for a certain time during cooling and experienced huge pressures because of Laplace compression. Pores, cavities, and the like in this state were expressed onto the particle surface. The only type of defects in NPs formed under extreme conditions were 

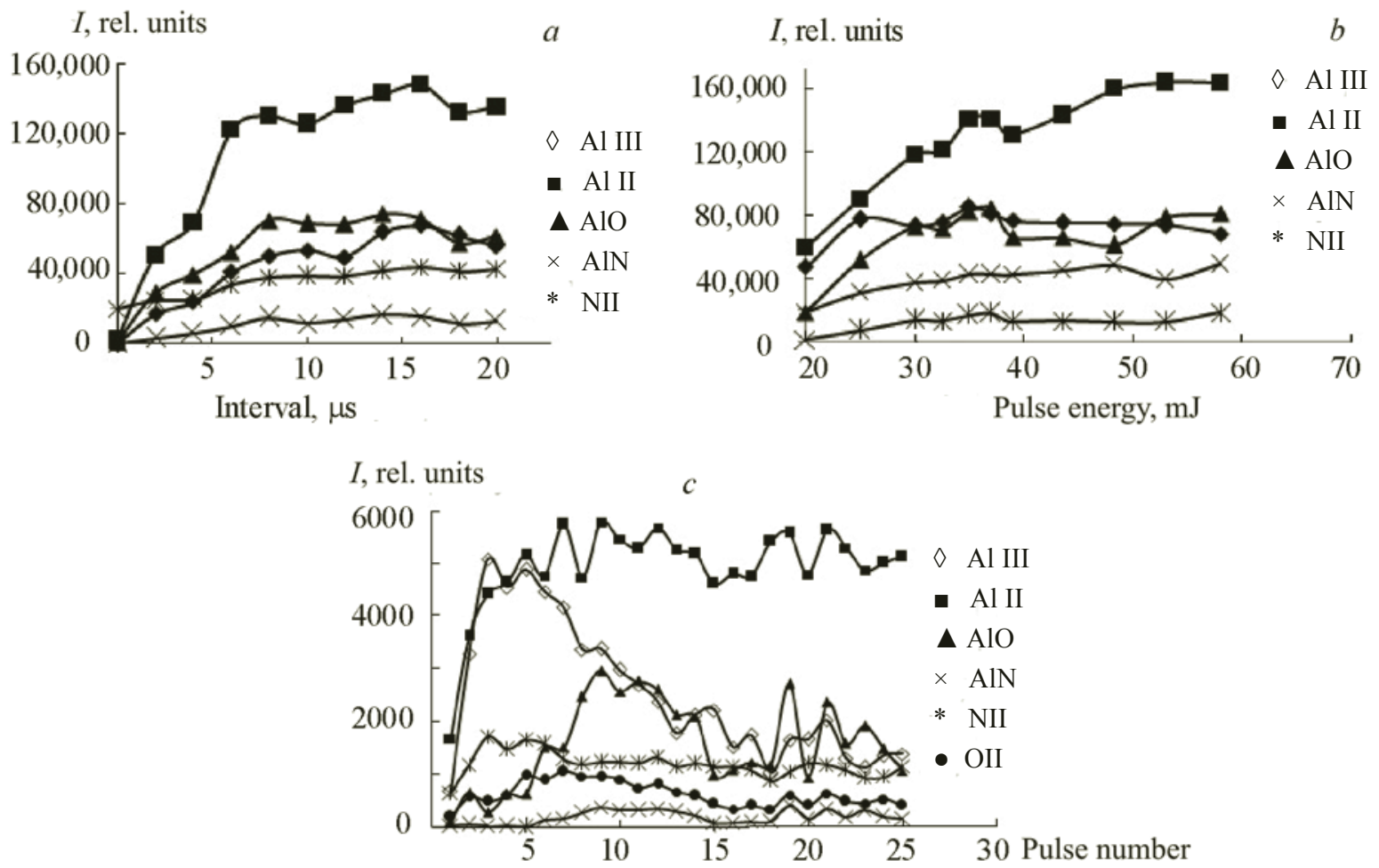

Fig. 1. Dependences of intensities of Al II (466.3 nm), Al III (452.92 nm), N II (399.5 nm), and O II lines $(407.8 \mathrm{~nm})$ and $\mathrm{AlO}(484.22 \mathrm{~nm})$ and AlN bands $(507.8 \mathrm{~nm})$ on the interval between pulses (a); pulse energy (b); and pulse number (c).
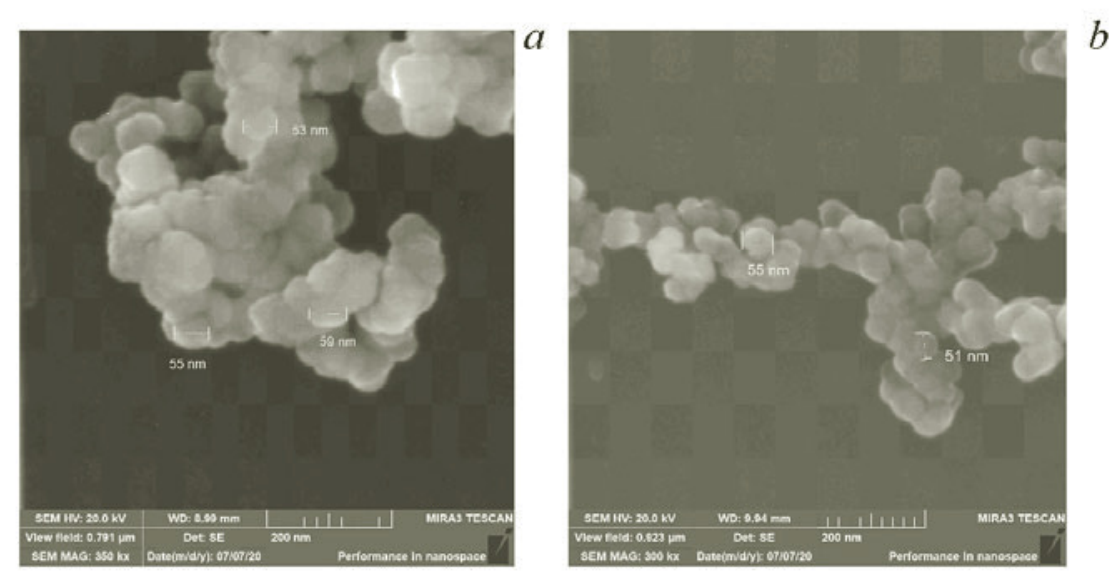

Fig. 2. SEM images of Al nanoparticles and agglomerates settled on the bottom (a) and side (b).

atomic shifts relative to the equilibrium positions. Agglomeration was due primarily to the thermodynamic instability of the metal NPs. The most important problem related to the size dependence of the surface tension was reported to be the thermodynamic stability of the NPs [14-16].

The particle distribution was affected by chemical reaction products, formation of particles of various compositions, complicated particle morphologies, and inevitable diffusion of components in the condensed phase. If two conditions occurred simultaneously, e.g., a rather high temperature [usually greater than $(2 / 3) T_{\mathrm{S}}$, where $T_{\mathrm{S}}$ is the melting point of bulk material] and strong interaction between particles, then coalescence occurred. The particles coalesced and lost their initial shape during the time of interparticle interaction and formed a single particle with a more equilibrium configuration. 
Coalescence of solid NPs occurred so fast at higher temperatures that it was reminiscent of the confluence of two liquid drops and was called liquid-like coalescence. If the NPs had a nonequilibrium shape and a very rough surface, then each particle was stretched and rounded, acquiring a more equilibrium shape, because of diffusion processes induced by surface tension forces. This process was called autocoalescence.

Particles that settled on the walls often stuck to each other, forming convoluted chains. The agglomerate chains were very difficult and sometimes practically impossible to separate. The particle surface was so active and clean that small particles could be sintered at temperatures below the melting point of the starting material. This meant that there was a low temperature limit to the production of very fine particles of the material. Thus, the actual finest particle that could be used in practice should always have a protective coating and/or be kept at lower temperatures.

Results and Discussion. Plasma formation in air was considerably complicated because of consequences that were practically unavoidable during formation of deep holes by double pulses. This caused ablated microparticles and clusters to accumulate in the atmosphere of the resulting holes. The action of a pulse following after a short time interval led to a lowthreshold optical breakdown of air saturated with metal microparticles and the appearance simultaneously of two plasma structures spread out in space. One of them was the ordinary laser plasma plume on the ablated surface and the bottom of the resulting hole; the other, a plasma dust cloud also generated on the laser-beam axis but displaced from the surface by a certain distance. In this instance, the appearance of the plasma dust cloud displaced from the surface by a certain distance led, on one hand, to additional shielding and, on the other, to the creation of a high-temperature high-pressure plasma cloud that scattered primarily along the hole direction.

The nonlinear dependence of the intensities of the AlO and AlN bands on energy could be explained as follows. Target mass was ejected and a channel was formed by the periodic pulsed high-intensity laser action on one point of the target because of laser ablation. The physical pattern of processes associated with channel formation was much more complicated than the situation where the laser radiation impinged on a smooth target surface. Concentration of the laser radiation in the channel caused absorption of the pulse energy to increase and, correspondingly, the temperature of the plasma in the channel and the efficiency of ion formation to increase. Also, a suspension or cloud containing target nanoand microparticles (clusters) could form in the channel after the first pulse. Interaction of the second pulse of laser radiation with this cloud in plasma-formation mode induced self-action, partial shielding, and problems with delivering the radiation energy to the channel bottom and, as a result, a change of shape of the channel [17-19].

Figure 3 shows dependences of microchannel shape and depth on pulse energy and number. The conic microchannel formed as the depth of the crater increased because the pulsed periodic laser action on the target (Fig. 3a) could act as a nozzle for more effective clustering of target matter as it passed through it. The concentration of the clusters and their distribution beyond the nozzle cross-section were determined by the nozzle geometry. Not all atoms, ions, or gas molecules clustered as the gas expanded through the nozzle but only a certain percent of them. The shape of the channel produced by 30 consecutive pulses of energy $37 \mathrm{~mJ}$ was observed to be more optimal for generating AlO radicals and AlN nanoclusters than that produced by fewer pulses.

The results were obtained at an energy density significantly greater than the shielding threshold. In this instance, the channel after 10 pulses was not deep enough for substantial accumulation of particles. The channel diameter was comparable to that of the beam waist. The stepped shape of the channel was indicative of the localization of the laser plasma in two regions, i.e., the end of the narrow part where only $\sim 10-20 \%$ of the incident radiation energy fell according to the obtained data and the end of the thickened part with a transition to narrowing where the remaining $80-90 \%$ of the energy in the plasma breakdown was stored and scattered. The regions were situated at a distance of $\sim 50-100 \mu \mathrm{m}$. Much of the incident radiation energy did not reach the bottom with this shape and was consumed for increasing the channel diameter by interacting with the side walls (Fig. 3b). This was especially noticeable if the pulse energy was increased to $53 \mathrm{~mJ}$.

Shielding with air breakdown on the particles was evident only if the aspect ratios of the channel (ratio of channel depth to diameter) reached $A>1$. This could be related to removal of particles from the crater at smaller $A$ because of convective air currents. New possibilities for this double-pulse action by series of consecutive pulses to form a plasma were reported [20]. A method for generating x-ray radiation with double-pulse laser action on a solid target under atmospheric conditions was proposed and demonstrated. Here, the first nanosecond laser pulse $\left(t_{\mathrm{p}}=30 \mathrm{~ns}, \lambda=308 \mathrm{~nm}, q=10^{8} \mathrm{~W} / \mathrm{cm}^{2}\right)$ initiated a surface optical breakdown that produced a zone of rarefied gas density for passage of the second (femtosecond high-intensity) laser pulse $\left(t_{\mathrm{p}}=110 \mathrm{fs}, \lambda=1.24 \mu \mathrm{m}, q=10^{16} \mathrm{~W} / \mathrm{cm}^{2}\right)$ when it reached the target. It was found that the experimental yield of hard $(E>2.5 \mathrm{keV}) \mathrm{x}$-ray radiation was greatest for an interval between pulses $t_{\text {int }}=10 \mu \mathrm{s}$. This delay corresponded to an increase in the yield of hard x-ray radiation in double-pulse (nano + femto) mode on the $\mathrm{Al}$ target as 
$a$

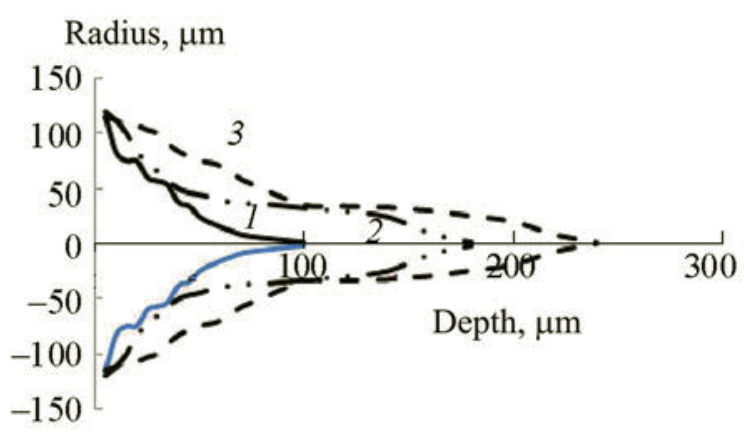

$b$

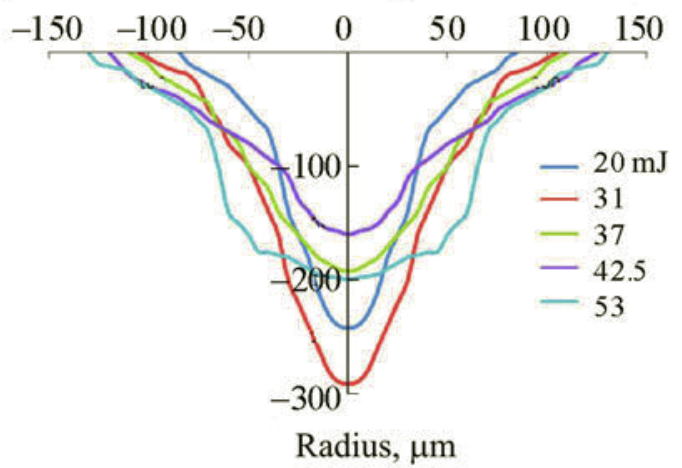

Radius, $\mu \mathrm{m}$

Fig. 3. Dependences of channel shape on pulse number: 10-10 (1), 20-20 (2), and 30-30

pulses (3) (a) and on energy (b).

compared to a single-pulse (femto) mode. The radiation increased up to 17 times on the target surface and up to 8 times in the channel as compared to the maximum yield of hard x-ray radiation from the action of single pulses. This indicated that the air density in the target surface region decreased after the action of the first pulse to 40 Torr and to 100 Torr in the channel.

Thus, the magnitude of the shielding depended on the repetition rate and energy of preceding laser pulses with multi-pulse action because ablated particles accumulated in the channel. The tine interval corresponding to restoration of the optical transparency of air in the beam waist was determined by the removal of particles from the channel or their settling on the walls. The minimum restoration time exceeded several seconds. The energy stored and scattered by the plasma breakdown did not help to increase the channel depth and was wholly transferred to the side walls, causing the linear ablation rate to stabilize over a broad range of active energy densities, because it was removed from the bottom. As a result, the channel diameter increased considerably through the action of the expanded and radiating plasma cloud and the radiation scattered by the plasma because of the action on the side walls.

The ejection velocity for typical laser pulse intensity of $q \approx 5 \cdot 10^{9} \mathrm{~W} / \mathrm{cm}^{2}$ was $3 \cdot 10^{6} \mathrm{~cm} / \mathrm{s}$; the plasma temperature in the second phase, $3 \cdot 10^{5} \mathrm{~K}[17,18]$. Considering the ejection rate and the average duration of the laser pulse irradiation (15 ns), a plasma plume length with one-dimensional expansion was $\sim 0.45 \mathrm{~mm}$ toward the end of this phase, which was comparable to the channel length. As a result, the plasma reached the sample surface during the time of action of the second laser pulse. It acquired a shape approaching a hemisphere with further ejection. The plasma region continued to expand after the action of the laser pulse subsided because of the energy and pressure stored in it. The expansion practically stopped after atmospheric pressure was reached within it. A sharply limited and relatively long-lived region of hot gas formed near the irradiated surface.

The degree of ionization of the laser plasma formed after the action of the second pulse on the condensation products was greater than that in the surface plasma. This was evident from the increase by practically an order of magnitude of the $\mathrm{Al}$ ion lines upon increasing the number of pulses. Naturally, the temperature of the laser plasma was significantly greater than that of the surface plasma.

The Ornstein method based on measuring the relative intensities of spectral lines belonging to a single element is one of the most common methods for determining plasma temperatures $[19,20]$. For example, plasma temperature can be calculated from the relative intensities of two atomic (or two ionic) lines with wavelengths $\lambda_{1}$ and $\lambda_{2}$ with known excitation energies $E_{1}$ and $E_{2}$, statistical weights of excited states, and radiative transition probabilities $(g A)_{1}$ and $(g A)_{2}$ :

$$
T=\frac{5040\left(E_{1}-E_{2}\right)}{\log (g A)_{1} /(g A)_{2}-\log \left(\lambda_{1} / \lambda_{2}\right)-\log \left(I_{1} / I_{2}\right)} \infty .
$$

Table 1 presents series of Al III ion lines and their parameters for determining the plasma temperature using the Ornstein method. The lines at $452.9 \mathrm{~nm}\left(E_{k 1}=20.55 \mathrm{eV}\right)$ and $360.16 \mathrm{~nm}\left(E_{k 2}=17.81 \mathrm{eV}\right)$ were selected to observe the optimum conditions for correct determination of the temperature by the Ornstein method $\left[k T \leq\left(E_{k 1}-E_{k 2}\right)\right][21,22]$. A formula for determining the laser plasma temperature in the region of existence of Al III ions is obtained by substituting the energies, statistical weights, and transition probabilities for the selected spectral lines: 

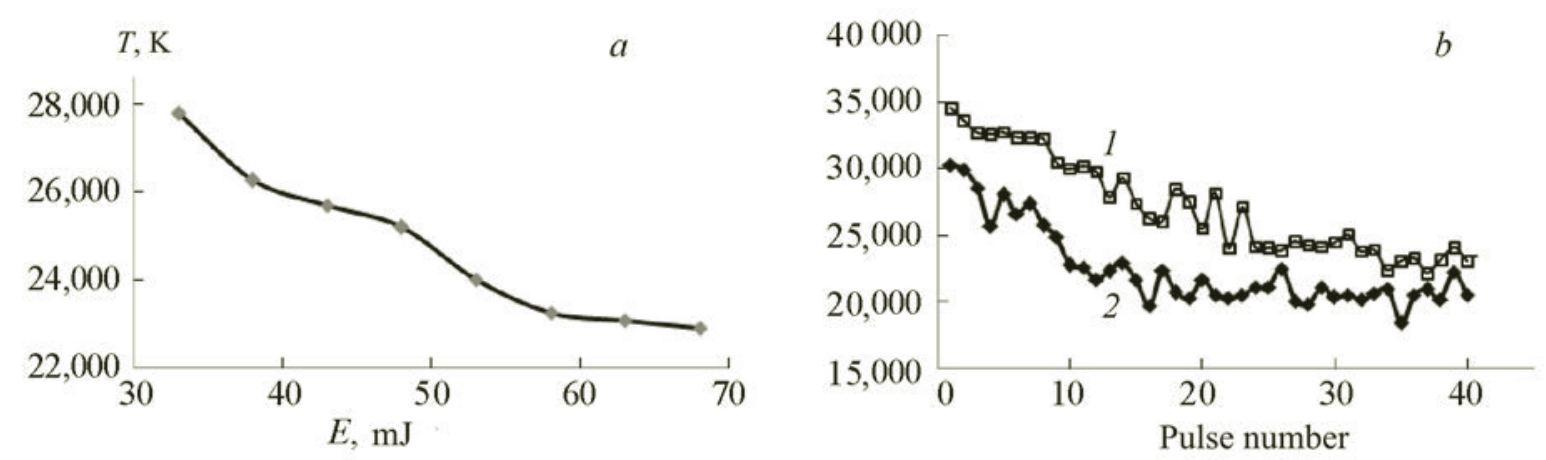

Fig. 4. Dependences of average laser plasma temperature in the Al III region on pulse energy (a) and pulse number for energy 33 (1) and $52 \mathrm{~mJ}$ (2) (b)

TABLE 1. Parameters of Al III Ion Lines

\begin{tabular}{|c|c|c|c|c|c|}
\hline$\lambda, \mathrm{nm}$ & $A_{k i} 10^{7}, \mathrm{~s}^{-1}$ & $E_{k}, \mathrm{~cm}^{-1}$ & $E_{i}, \mathrm{~cm}^{-1}$ & $g$ & $E_{k}, \mathrm{eV}$ \\
\hline 352.49 & 1.6 & 217,239 & 188,878 & 6,8 & \\
360.1628 & 13.1 & 143,713 & 115,957 & 4 & 17.81 \\
361.2356 & 14.5 & 143,633 & 115,958 & 2 & 17.8 \\
447.99 & & 189,928 & 167,613 & 8,10 & 23.54 \\
452.9 & 24.9 & 165,786 & 143,713 & 6 & 20.55 \\
\hline
\end{tabular}

$$
T=13,810 /\left(0.44-0.0995-\log \left(I_{1} / I_{2}\right)\right)=40,558-13,810 / \log \left(I_{1} / I_{2}\right) .
$$

Figure 4 shows dependences of the average laser plasma temperature in the Al III region on the pulse energy (series of 40 pulses) and pulse number in series for energies 33 and $52 \mathrm{~mJ}$. The temperature of this region estimated using these $\mathrm{Al}$ ion lines was $\sim 30,000-35,000 \mathrm{~K}$ for the first several pulses and then noticeably lower as the crater deepened. The average temperature for 40 pulses depended more weakly on the energy than for fewer pulses.

The plasma dust region at the exit from the micronozzle widened and was turbulently mixed. The temperature of the region dropped sharply. Because AlO emission appeared when the temperature was reduced to a certain value, it could be assumed that the temperature was too high until this moment and all molecules were dissociated (decomposition temperature of $\mathrm{AlO}$ is $4,400 \mathrm{~K}$ ). This was responsible for the increased formation rate of reaction products of $\mathrm{Al}$ atoms with air after formation of a rather deep microchannel $[12,13]$. The AlN content in the plasma was very low. Apparently, this was related to its lower thermal stability. AlN sublimes without melting at $2723 \mathrm{~K}$ [1].

Conclusions. Use of an interval between pulses of 7-16 $\mu$ s increased the concentration of $\mathrm{Al}$ ions and the products of their reaction with atmospheric $\mathrm{O}_{2}$ and $\mathrm{N}_{2}$ by several orders of magnitude as compared with no interval. The concentration of oxidized Al nanopowders reached a maximum for double-pulse ablation of a target by series of 15 consecutive double pulses and intervals between pulses $\Delta t \approx 7-16 \mu \mathrm{s}$. Interaction of a second pulse with condensation products formed in the channel after the action of the first pulse contributed most to the change of intensity of the $\mathrm{Al}$ spectral lines. The intensities of Al III ion lines decreased by $\sim 5$ times if the number of pulses in the series was increased above a certain level (10-20). This was related to active interaction of them with air molecules and atoms at the exit of the micronozzle and a shape change of the channel. The temperature in the plasma region with the maximum Al III content was estimated as $\sim 22,000-30,000 \mathrm{~K}$. $\mathrm{Al}$ nanopowders coated with a layer of $\mathrm{Al}_{2} \mathrm{O}_{3}$ produced via irradiation of an $\mathrm{Al}$ target by series of 15 double pulses per point had sizes of $50-60 \mathrm{~nm}$.

In general, the positive results of the conducted experiments should be considered a basis for further work on the improvement of the process for producing oxidized Al nanopowders and the products of their reaction with air. The 
product line of Al nanopowders mixed with metals and metal oxides, production of which presupposes chemical reaction of vaporized target particles with the surrounding gas, could also be substantially expanded.

\section{REFERENCES}

1. A. E. Sheidlin, A. Z. Zhuk, B. V. Kleimenov, E. I. Shkol'nikov, and M. Yu. Lopatin, Izv. Russ. Akad. Nauk, Energ., No. 2, 3-11 (2006).

2. M. K. Berner, V. E. Zarko, and M. B. Talavar, Fiz. Goreniya Vzryva, 49, No. 6, 1-28 (2013).

3. D. Sandaram, V. Yang, and V. E. Zarko, Fiz. Goreniya Vzryva, 51, No. 2, 37-63 (2015).

4. A. P. Il'in, O. B. Nazarenko, A. V. Korshunov, and L. O. Root, Features of the Physicochemical Properties of Nanopowders and Nanomaterials [in Russian], Tomsk Politekh. Univ., Tomsk (2012), pp. 12-15.

5. S. V. Matrenin, A. P. Il'in, L. O. Tolbanova, and E. V. Zolotareva, Izv. Tomsk. Politekh. Univ., 317, No. 3, $24-28$ (2010).

6. V. N. Snytnikov, Vl. N. Snytnikov, D. A. Dubov, V. I. Zaikovskii, A. S. Ivanova, V. O. Stoyanovskii, and V. N. Parmon, Prikl. Mekh. Tekh. Fiz., 48, No. 2, 172-184 (2007).

7. Yu. A. Kotov, V. V. Osipov, M. G. Ivanov, O. M. Samatov, V. V. Platonov, E. I. Azarkevich, A. M. Murzakaev, and A. I. Medvedev, Zh. Tekh. Fiz., 72, No. 11, 76-82 (2002).

8. S. V. Zabolotnov, A. A. Ezhov, L. A. Golovan', M. A. Lastovkina, V. I. Panov, V. Yu. Timoshchenko, and P. K. Kashkarov, Fiz. Tekh. Poluprovodn., 41, No. 8, 1017-1020 (2007).

9. V. G. Il'ves, Yu. A. Kotov, S. Yu. Sokovnin, and C. K. Rhee, Ross. Nanotekhnol., 2, Nos. 9-10, 96-101 (2007).

10. V. S. Burakov, A. F. Bokhanov, M. I. Nedel'ko, and N. V. Tarasenko, Kvantovaya Elektron. (Moscow), 33, No. 12, 1065-1071 (2003).

11. Kh. Bazzal, A. R. Fadaiyan, and A. P. Zazhogin, Zh. Belgosuniv., Fiz., No. 1, 34-42 (2017).

12. Kh. Bazzal, E. S. Voropai, A. P. Zazhogin, and M. P. Patanovich, in: Interinstitutional Collection of Scientific Works "Physicochemical Aspects of Studying Clusters, Nanostructures and Nanomaterials" [in Russian], No. 11, Tver' (2019), pp. 48-56.

13. Kh. Bazzal, N. A. Alekseenko, E. S. Voropai, M. N. Kovalenko, N. H. Trinh, and A. P. Zazhogin, in: Interinstitutional Collection of Scientific Works "Physicochemical Aspects of Studying Clusters, Nanostructures and Nanomaterials" [in Russian], No. 12, Tver' (2020), pp. 8-16.

14. V. M. Samsonov and N. Yu. Sdobnyakov, Poverkhn.: Rentgenovskie Sinkhrotronnye Neitr. Issled., No. 2, $73-78$ (2004).

15. N. Yu. Sdobnyakov, V. M. Samsonov, and A. N. Bazulev, Vestn. Novgorod. Gos. Univ. im. Yaroslava Mudrogo, Ser. Tekh. Nauki, No. 5 (103), 43-48 (2017).

16. N. A. Romanov, A. V. Nomoev, and S. V. Kalashnikov, Vestn. Buryat. Gos. Univ., No. 3, $93-99$ (2013).

17. S. M. Garnov, V. I. Klimentov, T. V. Konov, S. V. Kononenko, and F. Dausinger, Kvantovaya Elektron. (Moscow), 25, No. 1, 45-48 (1998).

18. S. M. Klimentov, S. V. Garnov, V. I. Konov, and T. V. Kononenko, Tr. Inst. Obshch. Fiz. im. A. M. Prokhorova, Ross. Akad. Nauk, 60, 13-29 (2004).

19. I. A. Bufetov, S. B. Kravtsov, and V. B. Fedorov, Kvantovaya Elektron. (Moscow), 23, No. 6, 535-540 (1996).

20. V. M. Gordienko, M. S. Dzhidzhoev, I. A. Zhvaniya, and I. A. Makarov, Kvantovaya Elektron. (Moscow), 37, No. 7, 599-600 (2007)

21. E. I. Kuznetsov and D. A. Shcheglov, Methods for Diagnosing High-Temperature Plasma [in Russian], Atomizdat, Moscow (1980), pp. 65-70.

22. Yu. A. Kuzyakov, K. A. Semenenko, and N. B. Zorov, Methods of Spectral Analysis [in Russian], MGU, Moscow (1990), pp. 36-40. 